Revista Brasileira de Meteorologia, v.27, n.1, 23 - 30, 2012

\title{
REDUÇÃO DE ESCALA DE DADOS DE VENTO DE MODELOS REGIONAIS PARA MODELOS DE DISPERSÃO ATMOSFÉRICA
}

\author{
HANS ROGÉRIO ZIMERMANN, OSVALDO LUIZ LEAL DE MORAES, CLAUDIO ALBERTO \\ TEICHRIEB, EVERSON DAL PIVA, VAGNER ANABOR
}

Universidade Federal de Santa Maria (UFSM), Santa Maria, RS, Brasil

zrhans@gmail.com, osvaldo.moraes@pq.cnpq.br, teichrieb@gmail.com, everson.bento@gmail.com,
vanabor@gmail.com

Recebido Junho 2010 - Aceito Agosto 2011

\begin{abstract}
RESUMO
Neste artigo apresenta-se o desenvolvimento de uma ferramenta para interpolar os dados meteorológicos oriundos de Modelos Regionais de Previsão de Tempo, ex. BRAMS, permitindo o acoplamento entre estes modelos e os modelos de dispersão atmosférica. Para a avaliação desta ferramenta, os campos de vento da saída do BRAMS são utilizados como dados de entrada no Modelo de Dispersão Atmosférica (Zimora), desenvolvido pelo Laboratório de Micrometeorologia da Universidade Federal de Santa Maria (UFSM).

Palavras chave: Modelo Dispersão Atmosférica, Modelo Regional, Assimilação de Dados.
\end{abstract}

\begin{abstract}
DOWNSCALING OF WIND DATA FROM REGIONAL MODEL FOR ATMOSPHERIC DISPERSION MODELS;

This paper discusses the development of a data interpolation scheme applicable to the regional numerical weather prediction models (i.e. BRAMS) output, allowing the coupling of these models with atmospheric dispersion models. To evaluate this tool, the BRAMS wind field is used as input to the Atmospheric Dispersion Model (Zimora) developed by the Laboratório de Micrometeorologia of the Universidade Federal de Santa Maria (UFSM).
\end{abstract}

Keywords: Atmospheric Dispersion Model, Regional Model, Data Assimilation

\section{INTRODUÇÃO}

A importância do monitoramento e controle da qualidade do ar, com o aumento da população e o rápido desenvolvimento industrial desde as últimas décadas, tem sido cada dia mais relevante, pois, os efeitos das emissões têm se mostrado mais alarmantes uma vez que, seus danos à sociedade e ao meio ambiente, não estão limitados apenas às áreas onde os poluentes estão sendo emitidos.

A estimativa da concentração de um poluente, emitido continuamente por uma fonte pontual e elevada, pode ser feita, hoje em dia, por uma variedade muito grande de modelos matemáticos. Muitos destes modelos numéricos de dispersão de poluentes ou também chamados de modelos de qualidade do Ar, ou ainda Modelos de Dispersão Atmosférica (MDA), têm sido desenvolvidos objetivando a investigação científica para dar suporte ao controle de emissões e à tomada de decisões no desenvolvimento de políticas ambientais (Seaman, 2000).

A qualidade dos resultados obtidos com modelos depende, tanto da complexidade deste MDA, quanto da certeza dos dados de entrada, onde esta última pode ser aprimorada através da assimilação de dados de medidas disponíveis. Contudo, justamente as principais deficiências atuais que ainda têm sido observadas, na maioria dos MDA, são as imprecisas estimativas da taxa de emissão de material pela fonte e a entrada de dados meteorológicos para a inicialização destes modelos. Outras deficiências não menos importantes, relacionadas à complexidade do MDA, consistem nas metodologias e limitações computacionais aplicadas às técnicas de resolução destes modelos (Kovalets et al., 2009).

Com relação a estas deficiências, a metodologia aqui desenvolvida procura até certo ponto minimizá-las, pelo menos no problema de entrada de dados; através da assimilação de dados meteorológicos. 
Esta metodologia consiste em uma técnica para redução de escala da saída de dados de modelos numéricos regionais de previsão de tempo, que considera-se neste caso como préprocessador meteorológico (PPM). Para tanto, o processo matemático de média ponderada é apropriado. Utilizando-se de argumentos heurísticos e procedimentos matemáticos, construiu-se um sistema de pesos multicamadas simples, para serem utilizados neste processo de média e para aumentar a baixa resolução da saída de dados do PPM, resultando em dados meteorológicos de alta resolução, que podem ser acoplados como dados de entrada em modelos de dispersão Eulerianos. Atualmente, estes modelos estão sendo amplamente utilizados, em sistemas de resposta de emergência, controle de qualidade do ar e em outros estudos de dispersão de poluentes pela comunidade científica.

A motivação do estudo desenvolvido neste artigo se deu da necessidade de quantificar as concentrações superficiais de poluentes, emitidas por fontes pontuais, considerando-se informações realistas da dinâmica da atmosfera local, durante o desenvolvimento e calibração de um modelo de dispersão de poluentes para rotineiramente ser usado como diagnóstico e prognóstico das concentrações superficiais de Material Particulado (MP), $\mathrm{NO}_{\mathrm{x}}$ e $\mathrm{SO}_{2}$, devido as emissões aéreas resultantes, por exemplo em um complexo Termoelétrico, da queima de carvão (Zimermann, 2009).

\section{METODOLOGIA}

Após algumas considerações literárias sobre Análise Objetiva, serão apresentados os processos do método desenvolvido que permite o acoplamento de dados do PPM, utilizando a versão brasileira do Sistema de Modelagem Atmosférica Regional (BRAMS) em operação na Universidade Federal de Santa Maria (UFSM).

Posteriormente, serão mostrados alguns resultados prévios da utilização desta técnica, através de gráficos resultantes de uma simulação utilizando MDA.

\subsection{Análise objetiva}

O problema de se relacionar valores esparsos a uma descrição organizada, como uma grade, já era conhecido desde o final da década de 40. Panofsky (1949) é considerado como um dos pioneiros em sugerir um método de análise conhecida como Análise Objetiva. Esta análise consiste no processo de transformar dados de observações em pontos irregularmente espaçados, em dados em pontos regularmente arranjados na forma de grade (Cressman, 1959).

Durante praticamente toda a década de 50 várias implementações foram efetuadas para se tentar evoluir neste tipo de análise, porém, uma de suas melhores técnicas era o método de ajuste polinomial por mínimos quadrados, mas este tinha a tendência de desenvolver alguns tipos de instabilidades em algumas áreas. Muitos esforços foram feitos para contornar este problema, até que ao final da década foi formada uma unidade conjunta de previsão numérica de tempo (JNWP) que, após extensivas tentativas de uso deste método, muda os procedimentos deste tipo de análise, seguindo métodos descritos por Bergthórsson e Döös apud Cressman (1959).

Estas mudanças foram feitas basicamente na metodologia de preparação dos dados, como coleta e remoção de erros, suavização e a utilização de uma interpolação através de médias ponderadas. Uma descrição clara deste procedimento pode ser encontrada no trabalho de Cressman (1959).

Na metade da década de 60, Barnes (1964) em seu trabalho apresenta o desenvolvimento de um esquema de interpolação por média ponderada convergente, para obter mais detalhes na análise de dados aleatoriamente espalhados. Nos anos anteriores, muitos estudos haviam sido feitos para encontrar uma boa técnica de interpolação para esquemas de análise objetiva, de maneira que mais detalhes fossem representados por esta análise. Até então, o melhor tipo de esquema, quer dizer, o mais bem sucedido, havia sido o esquema de ajuste de superfícies. Este esquema consistia em ajustar uma superfície geométrica aos dados conhecidos e calcular os valores determinados pela superfície, em quaisquer outros pontos de interesse. Especialmente pontos de grade.

Muitos outros autores como Dellert, Pfeffer et. al, Penn, Kunkel e Mount (1963) apud Barnes (1964) basearam seus trabalhos neste esquema. Na opinião destes, este método apresentava três principais desvantagens:

1. Os cálculos eram complicados e consumiam muito tempo de máquina para serem completados;

2. Os dados aos quais se ajustavam as superfícies eram escolhidos de maneira artificial (por permitirem melhores resultados);

3. O efeito de se utilizar dados errados podia ser catastrófico, uma vez que todos tinham o mesmo peso para obter a forma geral da superfície.

Para minimizar os efeitos indesejáveis, uma nova proposta surgiu na época, um processo de suavização foi recomendado, empregando o método de ajuste de mínimos quadrados das superfícies aos dados, onde a influência de cada dado tivesse um peso proporcional a sua distância em relação ao ponto de grade.

Este método foi considerado como uma combinação entre o método de ajuste de superfície (Barnes, 1964) e o método de interpolação por médias ponderadas proposta por (Cressman, 1959).

O método de médias ponderadas determinava o valor da variável em um ponto de grade, como sendo a soma de valores 
ponderados (com certa importância) de cada dado individual. Ou seja, quanto mais próximo um ponto estivesse do ponto de grade, maior seria a influência de seu valor.

A técnica de médias ponderadas, também já vinha paralelamente sendo utilizada, porém a maior desvantagem de tal esquema era sua tendência em "desuavisar" demais todas as pequenas variações no campo, fossem causadas tanto por erros de dados, quanto por distúrbios da atmosfera.

Em seu trabalho, Barnes (1964) descreveu uma técnica que recuperava os detalhes perdidos durante a aplicação do esquema de médias ponderadas. $\mathrm{O}$ fator peso usado em sua análise objetiva, foi desenvolvido da premissa fundamental de que uma distribuição bidimensional de uma variável atmosférica poderia ser representada por um número infinito de ondas harmônicas independentes, ou seja, uma representação integral de Fourier.

$\mathrm{Na}$ sua forma corrente, o fator de ponderação estava explicitamente relacionado à densidade de observações, que de forma final, dava a obtenção de resolução passível em qualquer análise escalar. Devido à dependência da densidade, aplicações de tal esquema eram restritos à regiões onde a distribuição de dados fosse razoavelmente uniforme, ou seja, a distância entre as estações de observação não se desviasse muito da média.

$\mathrm{Na}$ metade da década de 80 , os esquemas mais notáveis ainda eram os adotados por Barnes e Cressman. Em 1985, Hibbard e Wylie (1985), apresentam um método similar, cujos resultados são muito próximos ao de Barnes e Cressman, porém, permitindo um tempo de computação muito menor.

Este esquema está em uso até os dias de hoje, empregado no McIDAS. ${ }^{1}$

Nos métodos de Barnes (1964) e Cressman (1959), o tempo de computação para interpolar NS pontos de dados observados, em NG pontos de grade, é proporcional a NS*NG. No método proposto, esse tempo é proporcional a NS+NG, portanto, sendo executado cerca de 30 vezes mais rápido do que o Método de Barnes (1964) no McIDAS, quando houver, por exemplo, 1000 observações e 1500 pontos de grade (Hibbard e Wylie, 1985).

Este método consistia em escolher, para o peso a ser usado no esquema de médias ponderadas, uma função de uma

1. Do inglês: Man-Computer Interactive Data Access System (McIDAS), sistema de acesso de dados de interação homem-computador, é uma ferramenta pioneira de previsão de tempo, sendo uma coleção de programas e bibliotecas de usuários para visualização de dados geofísicos. Desenvolvida nos anos 70 , pelo centro de ciências e engenharia espaciais (SSEC) da universidade de Madison em Winsconsin (EUA), é usada continuamente até os dias de hoje. Reunindo as mais diversas fontes de informações como de satélites, estações, modelos numéricos e outros, é utilizada por vários setores como universidades e centros de pesquisas ao redor do mundo, agencia aeroespacial americana (NASA), Centro de Previsão de Tempestades (SPC) e alguns setores comerciais como empresas aéreas e de navegação. Considerada como uma ferramenta de sinergia que integra numerosos tipos de dados em um único sistema.

Fonte: http://www.ssec.wisc.edu/mcidas. classe restrita de tal modo que não fosse necessário calcular os produtos entre os pesos e os dados para cada par de pontos de grade e o ponto de observação. Para tal, eles aproveitavam do somatório e assim emulavam a técnica do filtro passabaixa de Barnes (1964), através da combinação das operações nas observações. A função peso que Hibbard e Wylie (1985) utilizaram foi um tipo de exponencial de meio-passo, que no caso unidimensional pode ser expressa por:

$$
w\left(x-x_{i}\right)=\left\{\begin{array}{lll}
e^{-\left(\frac{x-x_{i}}{r}\right)} & \text { se } & x_{i} \leq x \\
0 & \text { se } & x_{i}>x
\end{array}\right\}
$$

onde $\mathrm{r}$ é uma constante que determina o peso da função ou suavização espacial do dado.

O ponto chave do esquema de Barnes (1964) consistia em dividir a expressão acima em duas partes, dois somatórios, sendo este o segredo de sua velocidade.

Este método de Hibbard e Wylie (1985) se aproximava muito bem do método proposto por Barnes onde havia dados. A única situação onde o método diferia bastante era em áreas onde havia grande ausência de dados, e as informações tinham que ser interpoladas sobre longas distâncias. Tais diferenças surgiam porque não se pode perfeitamente aproximar uma exponencial de distância quadrática, como a proposta por Barnes (1964), em uma soma de exponenciais lineares, ou seja, a comparação entre os resultados eram muito bons para pequenas distâncias, mas se desviavam muito quando as distâncias eram muito grandes. Nestes casos, no método de Hibbard e Wylie (1985), eles atribuíam um peso levemente maior aos dados extrapolados por causa desta aproximação.

Em áreas aonde não existiam dados, na realidade não havia informação na análise uma vez que a física da variável não era considerada. Por estas razões, Hibbard e Wylie (1985) sustentaram que seu esquema de análise era mais preciso do que o esquema de Barnes (1964) e Cressman (1959), e poderia substituí-los.

\subsection{Esquema de interpolação}

O MDA desenvolvido pelo Laboratório de Micrometeorologia (LuMet-UFSM), executa sua simulação de dispersão atmosférica, com uma resolução espacial maior do que a disponível pelo seu PPM. Então, foi necessário desenvolver um esquema para aumentar a resolução espacial dos dados de entrada. Para tanto, foi desenvolvido um esquema múltiníveis, baseado em médias ponderadas apropriadas para utilizar no MDA, aumentando a baixa resolução do PPM em qualquer resolução espacial múltipla necessária.

A concepção dos métodos de Cressman (1959) e as propostas de Hibbard e Wylie (1985) funcionam muito bem, 
quando aplicadas nos casos em que as observações estão irregularmente espaçadas, porém no caso de observações regularmente espaçadas (advindas de PPM), estes métodos não são tão efetivos, pois há um custo computacional desnecessários. Além disto, as aproximações subestimam certos valores quando aplicados em interpolação de quatro pontos como este caso.

O método proposto é muito semelhante ao de Hibbard e Wylie (1985), apenas difere na concepção da expressão que será utilizado como função peso $(w)$ na interpolação com médias ponderadas.

Para conceber esta expressão, por simplicidade serão considerados apenas dois pontos A e B, representando dois valores conhecidos de uma quantidade escalar qualquer, espaçados por uma distância $\mathrm{D}$ entre eles, representando baixa resolução. Visando aumentar esta resolução por um fator d. É possível resolver qualquer novo ponto entre eles utilizando a técnica de médias ponderadas, atribuindo para A e B pesos específicos em cada novo subintervalo $(\mathrm{D} / \mathrm{d})$ na nova escala entre A e B.

A expressão matemática para média ponderada é dada por:

$$
\bar{x}_{n}=\frac{\sum_{i=1}^{m} x_{i} w_{i}}{\sum_{i=1}^{m} w_{i}}
$$

onde xi é a entidade geradora (valor de A ou B), m é o número de pontos com valores conhecidos, dois neste exemplo (A e B), sendo interpolados e $w_{i}$ é o peso associado a cada intervalo onde deseja-se resolver.

Para determinar os pesos de A e B, do exemplo acima, é necessário definir duas hipóteses:

1. Do ponto de vista de A, seu peso deverá contribuir ao máximo em sua própria posição, e contribuir ao mínimo na posição do ponto $\mathrm{B}$;

2. Do ponto de vista de B, a regra é a mesma, sua contribuição será máxima em sua própria posição e mínima na posição do ponto A.

Com estas suposições, é possível formular uma expressão matemática obedecendo tais suposições, que tenha um papel de função peso tanto aos pesos de $\mathrm{A}$ como de $\mathrm{B}$, de tal maneira que não afete os valores de $\mathrm{A}$ e $\mathrm{B}$ em suas próprias posições e permita a interpolação entre qualquer ponto entre eles.

Generalizando para quatro valores conhecidos, que a partir de agora podem se chamar observações, $U_{A}, U_{B}, U_{C}$ e $U_{D}$, igualmente espaçados como na Figura 1, é possível expressar estes pesos como:

$$
\begin{aligned}
& H_{i, j}^{U_{A}}=\left(\frac{p-\max (i, j)}{\delta g}\right) \\
& H_{i, j}^{U_{D}}=1-\left(\frac{p-\min (i, j)}{\delta g}\right)
\end{aligned}
$$

$$
\begin{aligned}
& H_{i, j}^{U_{B}}=H_{p+1-i, j}^{U_{D}} \\
& H_{i, j}^{U_{C}}=H_{j, i}^{U_{B}}
\end{aligned}
$$

Nas Equações 2-5 os índices i, j representam o nó da grade com a nova escala de resolução que se está construindo, $\delta g$ é o fator de escala espacial que se está reduzindo a grade inicial, $p=\delta g+1$ e H representa a função peso para seu respectivo ponto conhecido, denotado pelo sobrescrito, em cada novo nó da grade.

Esse conjunto de equações, ao serem iterados nos índices $\mathrm{i}, \mathrm{j}$, se tornarão matrizes cujos elementos farão o papel dos pesos $w_{i}$ na Equação 1, que pode ser generalizada para duas dimensões como:

$$
\widetilde{Z}_{i, j}=\frac{\sum_{k=1}^{m} U_{k} H_{i, j}^{U_{k}}}{\sum_{k=1}^{m} H_{i, j}^{U_{k}}} .
$$

$\mathrm{Na}$ Equação 6 o índice $\mathrm{k}$ representa cada um dos valores observados $U_{A}, U_{B}, U_{C}$ e $U_{D}$, e resolvido para $i, j$ na faixa de $\mathrm{p}=1$ a $p=\delta g+1$, é obtido uma matriz $\widetilde{Z}_{5 x 5}$ que representa os novos nós para a resolução desejada.

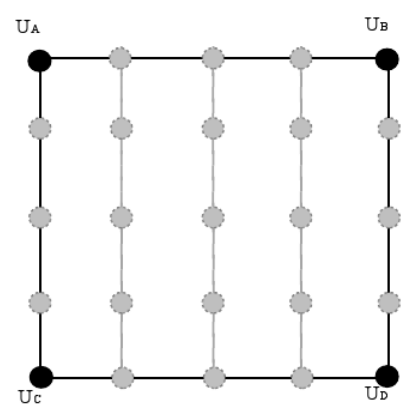

Figura 1 - Exemplo de uma grade bidimensional com quatro nós de valores conhecidos (preto) e nós que precisam ser apropriadamente interpolados, para se obter seus valores (cinza) em uma resolução maior.

O fluxograma da Figura 2 representa de forma esquemática a entrada de dados oriundos do PPM BRAMS e seus passos no processamento de assimilação e construção de uma nova grade com alta resolução.

De uma maneira prática, o MDA assimila o campo de vento, obtido da saída do PPM, com resolução espacial de $20 \mathrm{~km}$. Então, através do método descrito, ele aumenta esta resolução aplicando uma redução de escala para $5 \mathrm{~km}$. A Figura 1 mostra uma parcela de uma grade onde o MDA efetua suas simulações. Os quatro pontos pretos simbolizam os valores observados (obtidos do PPM) e os pontos em cinza representam os valores assimilados para a nova resolução espacial, obtidos através de esquema de interpolação.

Tendo a Figura 2, como exemplo do esquema de entrada de dados no MDA assimilando um campo de velocidade do 


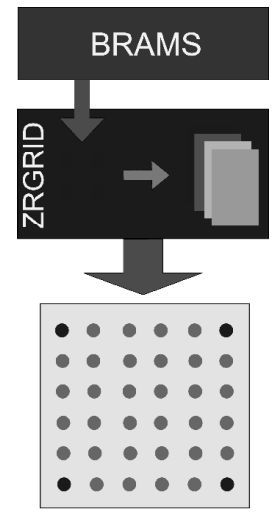

Figura 2 - Fluxograma esquemático do módulo (ZRGRID) do Zimora que reduz a escala do campo de vento, gerado pela saída operacional do BRAMS, através da interpolação.

vento de um modelo numérico de previsão de tempo PPM, como observações, pode-se demonstrar um exemplo prático com os resultados da Seção 3.

\section{RESULTADOS E DISCUSSÃO}

\subsection{Obtenção do pesos}

Considere uma célula, como na Figura 1, com os pontos em preto simbolizando as observações $\mathrm{U}_{\mathrm{A}}=3.629, \mathrm{U}_{\mathrm{B}}$ $=3.630, \mathrm{U}_{\mathrm{C}}=4.025$ e $\mathrm{U}_{\mathrm{D}}=3.994$, representando a velocidade do vento em m/s em uma resolução espacial de $20 \mathrm{Km}$. Para resolver os pontos (cinza) com uma nova resolução de grade espacial de $5 \mathrm{~km}$, o MDA primeiramente deve encontrar o fator de escala espacial para sua redução:

$$
\delta g=\frac{20 k m}{5 k m}=4
$$

Aplicando este valor nas Equações 2 a 5 e integrando i, $\mathrm{j}$ de $\mathrm{p}=1$ a $p=\delta g+1$, serão obtidas as $\mathrm{p}-1$ matrizes $\widetilde{H}_{p x p}$, que serão chamadas de camadas, cujos valores sãomostradosnas Tabelas 1 a 5 .

Calculando a primeira camada com os pesos para a observação $U_{\mathrm{A}}$ em cada novo ponto de grade, são obtidos os valores mostrados na Tabela 1:

Tabela 1 - Valores para a primeira camada de pesos $\widetilde{H}_{5 x 5}^{U_{A}}$.

\begin{tabular}{|l|l|l|l|l|}
\hline 1.000 & 0.750 & 0.500 & 0.2500 & 0.000 \\
\hline 0.750 & 0.750 & 0.500 & 0.2500 & 0.000 \\
\hline 0.500 & 0.500 & 0.500 & 0.2500 & 0.000 \\
\hline 0.250 & 0.250 & 0.250 & 0.2500 & 0.000 \\
\hline 0.000 & 0.000 & 0.000 & 0.0000 & 0.000 \\
\hline
\end{tabular}

Calculando a segunda camada com os pesos para a observação $U_{B}$ em cada novo ponto de grade, são obtidos os valores mostrados na Tabela 2.

Calculando a terceira camada com os pesos para a observação $U_{\mathrm{C}}$ em cada novo ponto de grade, são obtidos os valores mostrados na Tabela 3 .

Finalmente calculando a quarta camada com os pesos para a observação $U_{D}$ em cada novo ponto de grade, são obtidos os valores mostrados na Tabela 4:

Utilizando essas camadas dentro da Equação 6, sendo $\mathrm{k}$ relativo a cada observação $U_{A}, U_{B}, U_{C}$ e $U_{D}$, iterando $i, j$ de $p$ $=1$ a $p=\delta g+1$ será obtida a camada representada pela matriz e $\widetilde{Z}_{5 x 5}$, cujos elementos serão os novos valores para os nós com resolução espacial de $5 \mathrm{~km}$ mostrados na Tabela 5:

O mesmo procedimento é aplicado, repetido, até o último ponto de grade do PPM, sempre em grupos de quatro observações. A generalização pode ser aplicada na vertical, mudando apenas a resolução espacial tanto no PPM, quanto na grade do MDA, tal que no final do processo ele terá construído uma grade com hiper-células de volume $5 \mathrm{~km}$ x $5 \mathrm{~km}$ x $100 \mathrm{~m}$.

Tabela 2 - Valores para a segunda camada de pesos $\widetilde{H}_{5 x 5}^{U_{B}}$.

\begin{tabular}{|l|l|l|l|l|}
\hline 0.000 & 0.250 & 0.500 & 0.750 & 1.000 \\
\hline 0.000 & 0.250 & 0.500 & 0.750 & 0.750 \\
\hline 0.000 & 0.250 & 0.500 & 0.500 & 0.500 \\
\hline 0.000 & 0.250 & 0.250 & 0.250 & 0.250 \\
\hline 0.000 & 0.000 & 0.000 & 0.000 & 0.000 \\
\hline
\end{tabular}

Tabela 3 - Valores para a terceira camada de pesos $\widetilde{H}_{5 x 5}^{U_{C}}$

\begin{tabular}{|l|l|l|l|l|}
\hline 0.000 & 0.000 & 0.000 & 0.000 & 0.000 \\
\hline 0.250 & 0.250 & 0.250 & 0.250 & 0.000 \\
\hline 0.500 & 0.500 & 0.500 & 0.250 & 0.000 \\
\hline 0.750 & 0.750 & 0.500 & 0.250 & 0.000 \\
\hline 1.000 & 0.750 & 0.500 & 0.250 & 0.000 \\
\hline
\end{tabular}

Tabela 4 - Valores para a quarta camada de pesos $\widetilde{H}_{5 x 5}^{U_{D}}$

\begin{tabular}{|l|l|l|l|l|}
\hline 0.000 & 0.000 & 0.000 & 0.000 & 0.000 \\
\hline 0.000 & 0.250 & 0.250 & 0.250 & 0.250 \\
\hline 0.000 & 0.250 & 0.500 & 0.500 & 0.500 \\
\hline 0.000 & 0.250 & 0.500 & 0.750 & 0.750 \\
\hline 0.000 & 0.250 & 0.500 & 0.750 & 1.000 \\
\hline
\end{tabular}


Tabela 5 - Valores finais da grade do MDA com o campo de velocidades do vento com em alta resolução, interpolados pelo esquema de multicamadas de médias ponderadas. Camada $\widetilde{Z}_{5 x 5}$.

\begin{tabular}{|l|l|l|l|l|}
\hline 3.629 & 3.629 & 3.630 & 3.630 & 3.630 \\
\hline 3.728 & 3.756 & 3.756 & 3.757 & 3.721 \\
\hline 3.827 & 3.822 & 3.820 & 3.817 & 3.812 \\
\hline 3.926 & 3.888 & 3.883 & 3.878 & 3.903 \\
\hline 4.025 & 4.017 & 4.010 & 4.002 & 3.994 \\
\hline
\end{tabular}

\subsection{Aplicação para simulação real}

Nas Figuras 3 a 5, são apresentados alguns exemplos do resultado das simulações executadas pelo modelo de dispersão atmosférica desenvolvido. O dia escolhido para a demonstração não possui nenhuma finalidade especial, apenas é utilizado para fins de exemplificação.

Os primeiros testes de simulação, utilizando um campo de vento de baixa resolução $(20 \mathrm{~km})$, exatamente como se encontra hoje a saída operacional do modelo regional de previsão numérica da UFSM, são mostrados pelas Figuras de 3 a 7.

Em termos de comparação, as Figura 8 até Figura 12 mostram as mesmas simulações. Nestas, aplica-se a metodologia descrita neste artigo $(5 \mathrm{~km})$; junto à forma da pluma, estão representados os vetores indicando a magnitude e direção do vento a cada simulação. Com esta representação pode-se observar a boa concordância de deslocamento da pluma na simulação. Em ambos os casos, as unidades não estão adequadamente dimensionadas à realidade, pois estão expressas em unidades de iteração, ou seja, a emissão de uma unidade de concentração a cada interação. $\mathrm{O}$ valor real da concentração simulada pode ser calculado (ajustado à ferramenta) sabendo-se a taxa de emissão da fonte. O objetivo destas figuras é apenas ilustrar que o uso da ferramenta para acoplamento do campo de vento, através da redução de escala, não alterou a forma e comportamento de movimento da pluma ao decorrer do tempo a cada simulação, apenas aumentado sua resolução.

Observa-se em todos os casos, que a aplicação e utilização do esquema proposto suavizam significativamente a forma da pluma de concentração. Esta suavização se deve à maior resolução, obtida através da redução sobre os dados do campo de vento e coeficientes de difusividades do PPM (BRAMS).

\section{CONCLUSÃO}

A metodologia desenvolvida se mostrou adequada em minimizar as deficiências, na questão do problema de entrada de dados, através do acoplamento de dados meteorológicos.

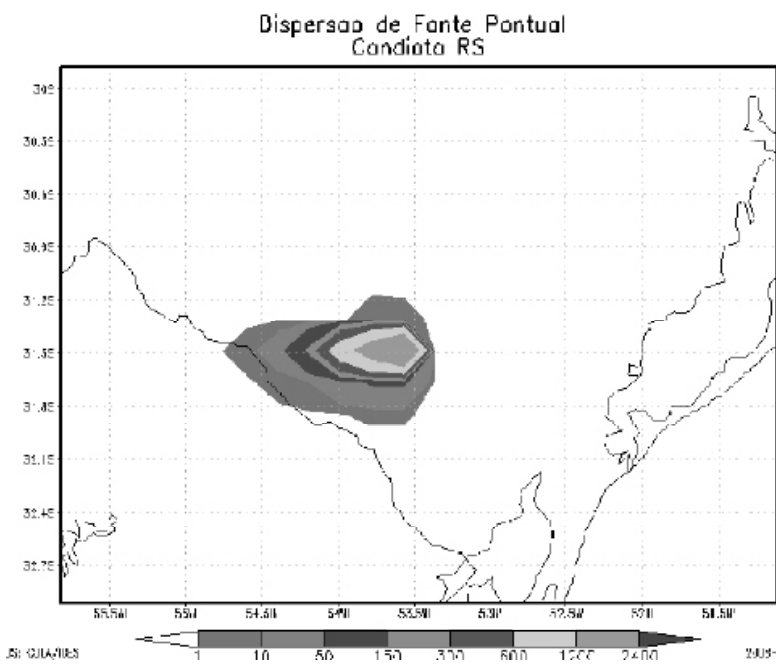

Figura 3 - Simulação de 1 h de dispersão, utilizando campo de vento de baixa resolução.

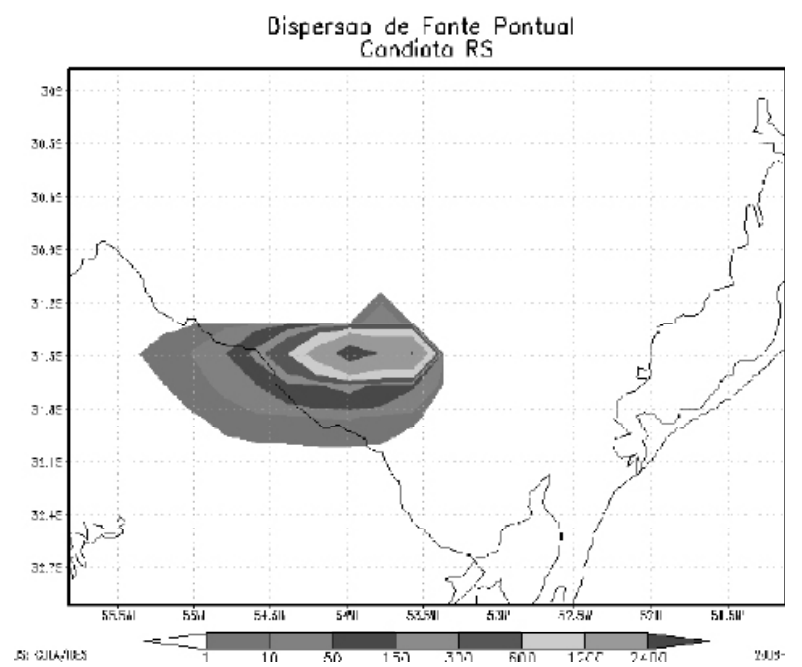

Figura 4 - Simulação de $2 \mathrm{~h}$ de dispersão, utilizando campo de vento de baixa resolução.

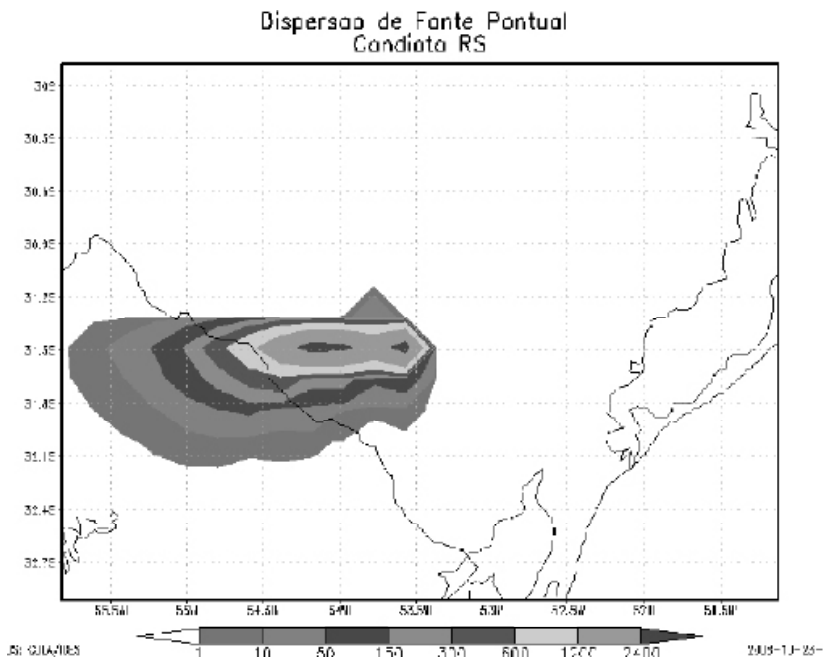

Figura 5 - Simulação de 3 h de dispersão, utilizando campo de vento de baixa resolução. 


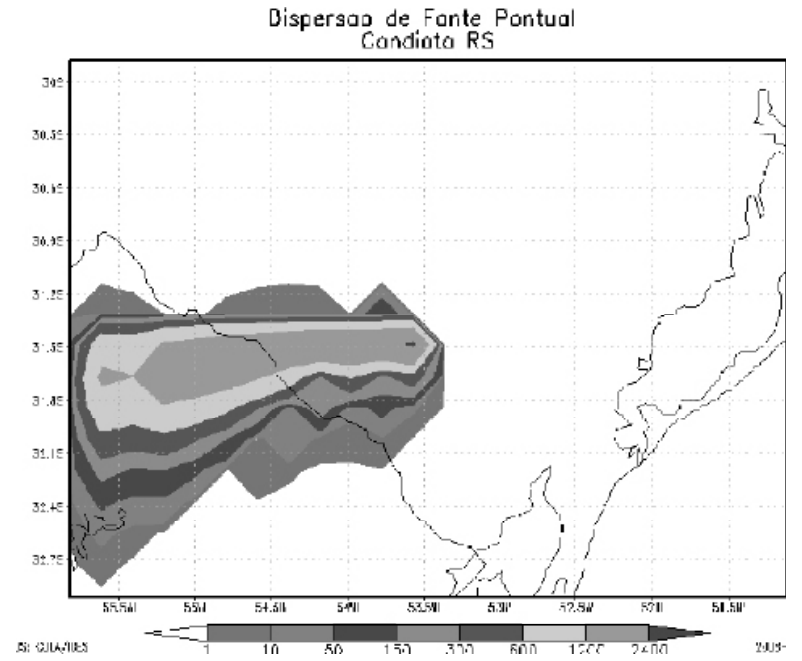

Figura 6 - Simulação de 6 h de dispersão, utilizando campo de vento de baixa resolução.

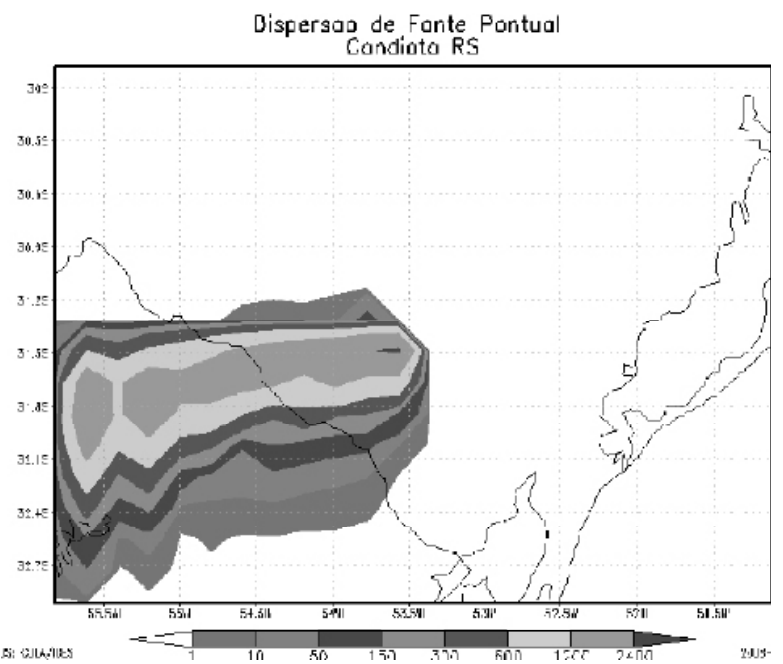

Figura 7 - Simulação de 7 h de dispersão, utilizando campo de vento de baixa resolução.

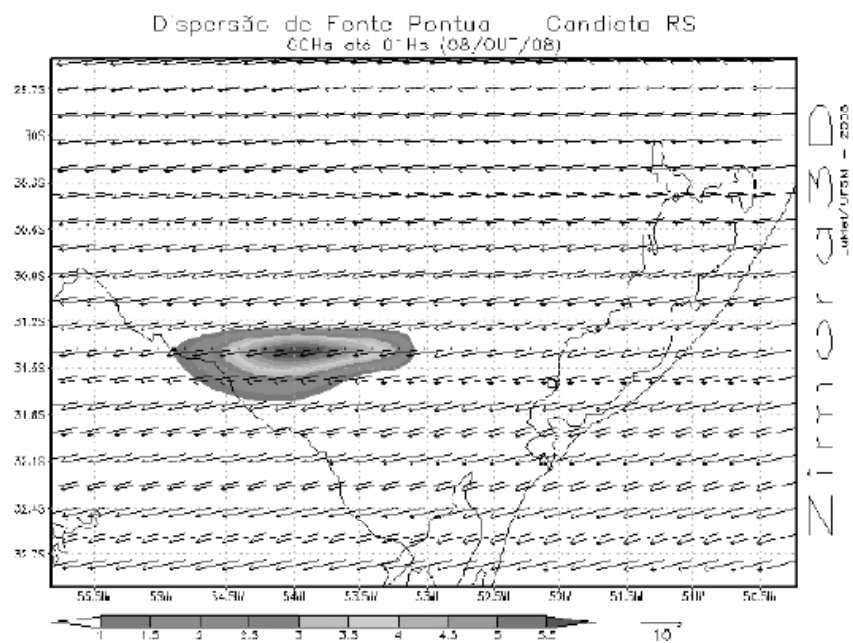

Figura 8 - Simulação de 1h de dispersão, utilizando campo de vento de alta resolução.

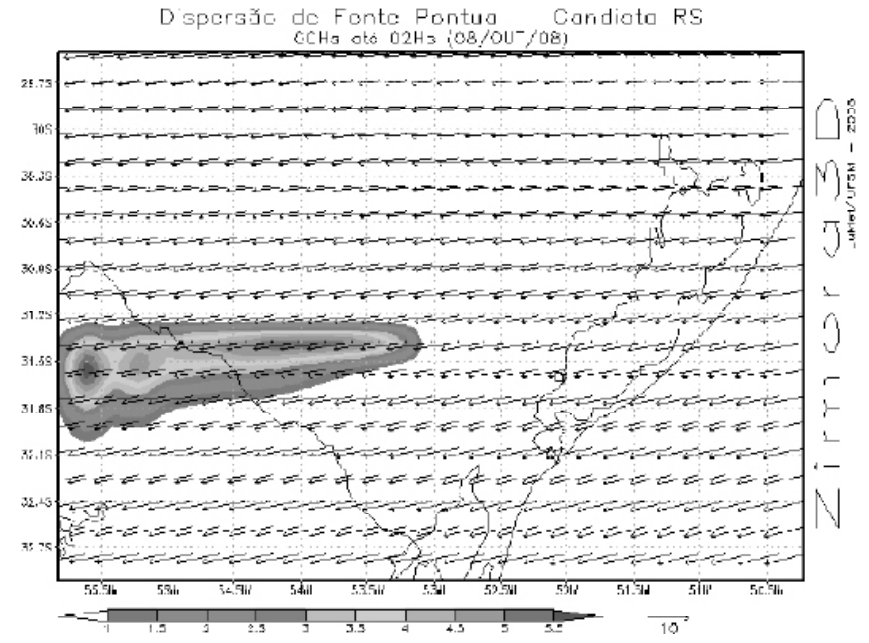

Figura 9 - Simulação de $2 \mathrm{~h}$ de dispersão, utilizando campo de vento de alta resolução.

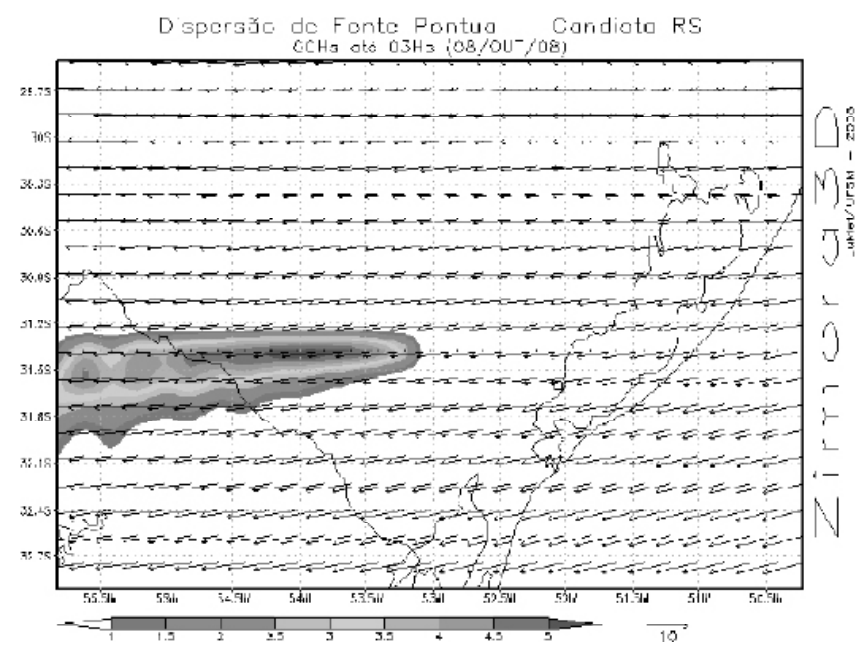

Figura 10 - Simulação de $3 \mathrm{~h}$ de dispersão, utilizando campo de vento de alta resolução.

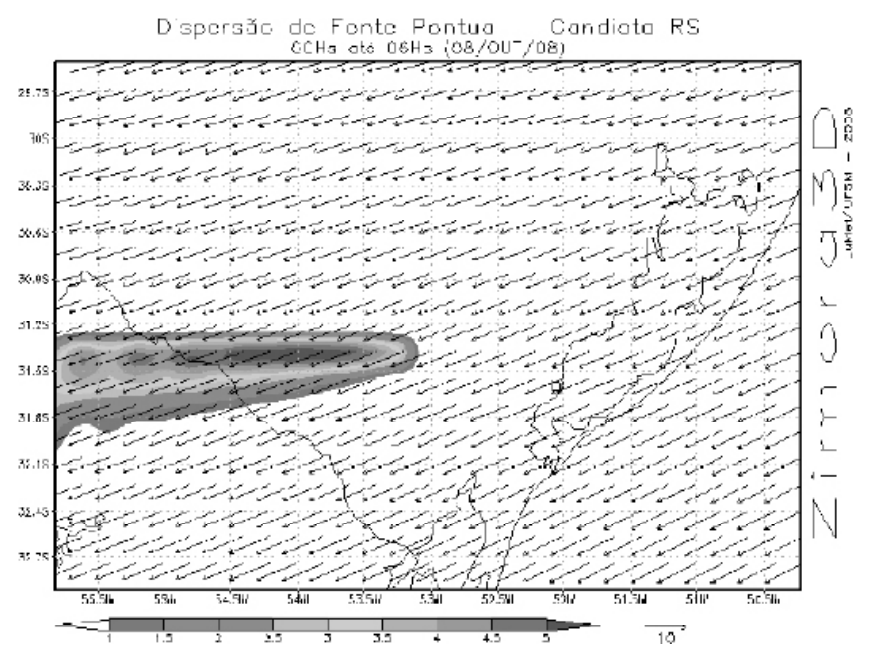

Figura 11 - Simulação de 6 h de dispersão, utilizando campo de vento de alta resolução. 


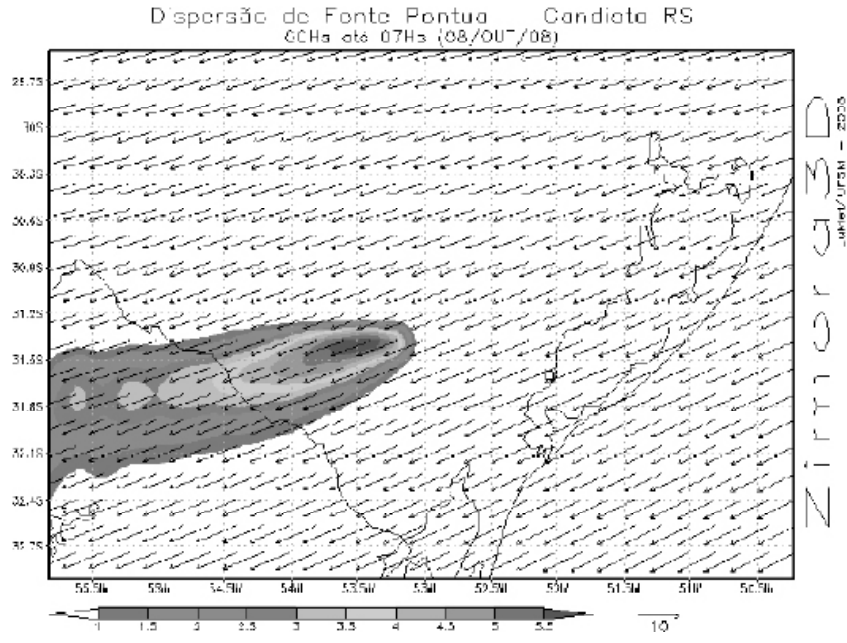

Figura 12 - Simulação de 7h de dispersão, utilizando campo de vento de alta resolução.

O esquema de interpolação mediante o uso do processo matemático de média ponderada, através da construção de um sistema de pesos multicamadas, mostrou-se bastante eficiente e resultou numa boa aproximação para se obter campo de vento com maior resolução através da redução de escala da saída de dados de modelos numéricos regionais de previsão de tempo, considerado neste caso como pré-processador meteorológico (PPM).

Este esquema permite que as equações, empregadas na resolução dos processos de transporte (Zimermann, 2009), tenham suas aproximações mais apuradas, conseqüentemente melhorando a qualidade e confiabilidade da simulação.

\section{AGRADECIMENTOS}

Os autores agradecem ao Conselho Nacional de desenvolvimento Científico e Tecnológico (CNPq), UFSM, Instituto Nacional de Pesquisas Espaciais (INPE) e Companhia de Geração Térmica de Energia Elétrica CGTEE, pelo financiamento e apoio. Ao Grupo de Modelagem Atmosféricas (GruMA) pela disponibilização dos dados do BRAMS.

\section{REFERÊNCIAS}

BARNES, L. S. A Technique for maximizing Details in Numerical Weather Map Analysis. Journal of Applied Meteorology, Washington/US, v.3, p.396-409. 1964.

CRESSMAN, G. P. An Operational Objective Analysis System. Monthly Weather Review, Washington/US, v.87, p.367374. 1959.

HIBBARD, W. L.; WYLIE, D. P. An Efficient Method of Interpolating Observations to Uniformly Spaced Grids. In: Conf. Interactive Information and Processing systems (IIPS) for Meteorology, Oceanography and Hydrology. 1985.

KOVALETS, I. V.; TSIOUTI, V.; ANDROPOULOS, S.; BARTZIS, J. G. Improvement of Source and Wind Field Input of Atmospheric Dispersion Model by Assimilation of Concentration Measurements: Method and Applications in Idealized Settings. Applied Mathematical Modeling, v.33, n.8, p.3511-3521. 2009.

PANOFSKY, H. A. Objective Weather Map Analysis. Journal of Meteorology, Lancaster/US, n.6, v. 6, p.386-392. 1949,

SEAMAN, L. N. Meteorological Modeling for Air-Quality Assessments. Atmospheric Environment, n.34, p.22312259. 2000,

ZIMERMANN, H. R. Zimora: um modelo numérico 3D de dispersão atmosférica. 2009. 197 f. Tese (Doutorado em Física) - Universidade Federal de Santa Maria Santa Maria, Santa Maria, 2009. 